\title{
Power Efficient Time-Division Random-Access Model Based in Wireless Communication Networks
}

\author{
Dr. V. Suma \\ Professor, \\ Department of Information Science \& Engineering, \\ Dayananda Sagar College of Engineering, \\ Bangalore, India.
}

\begin{abstract}
The conventional infrastructure for mobile-communication is used for providing internet-of-things (IoT) services by the third-generation partnership project (3GPP) with the help of the recently developed cellular internet-of-things (CIoT) scheme. Random-access procedure can be used for connecting the large number of IoT devices using the CIoT systems. This process is advantages as the huge devices are accessed in a concurrent manner. When random access procedures are used simultaneously on a massive number of devices, the probability of congestion is high. This can be controlled to a certain extent through the time division scheme. A power efficient time-division random access model is developed in this paper to offer reliable coverage enhancement (CE) based on the coverage levels (CL). The quality of radio-channel is used for categorization of the CIoT devices after assigning them with CLs. The performance of random-access model can be improved and the instantaneous contention is relaxed greatly by distributing the loads based on their coverage levels into different time periods. Markov chain is used for mathematical analysis of the behavior and state of the devices. The probability of blocking access, success rate and collision control are enhanced by a significant level using this model in comparison to the conventional schemes.
\end{abstract}

Keywords: Collision, time division, power efficient, coverage enhancement, coverage level, cellular internet-of-things;

\section{Introduction}

Telematics, healthcare, smart metering and various other services that are ubiquitous and innovative can make use of Internet-of-things (IoT) [1]. Highly distinguished characteristics are offered by the IoT services in contrast to the conventional communication schemes. Low energy and low cost must be maintained while measuring the location or collecting messages from IoT systems and devices to offer IoT services. When ZigBee, $\mathrm{Wi}-\mathrm{Fi}$, Bluetooth and other short range communication technologies are used, the low-power IoT device manufacturing cost is reduced. However, this is applicable only for lesser driver count [2]. Further, the capital expenditure is high as the system infrastructure deployment is dense due to the need for accommodation of all IoT devices in the wide coverage area. Low power long-range technologies are to be developed to improve the efficiency of IoT systems. Unlicensed bands are used in designing private models using SigFox and Long Range (LoRa) projects for IoT systems. The IoT services are offered by the radio resources for lesser cost as the solutions are of unlicensed bands. The unavailability of facilities and infrastructure leads to substantial consumption of financial resources and time for establishing new IoT systems [3].

The third-generation partnership project (3GPP) enables retaining the conventional cellular networks for CIoT applications due to the cost and time expenses [4]. The long-term evolution (LTE) and LTE-Advanced platform based architectures namely general packet radio service (GPRS) and global system for mobile communication (GSM) platforms are used for developing a clean-state architecture in coordination with 3GPP. Several technologies like the narrowband-IoT (NB-IoT) and extended coverage-GSM (EC-GSM) has been developed as evolved LTE and clean-state technologies respectively [5]. The massive IoT devices are connected with high efficiency random-access schemes that act as a deciding parameter for the CIoT system performance. The evolved NodeB acts as a single access point that may be used for interconnection of over 50,000 IoT devices using 3GPP CIoT services based on its high-level objectives. When compared to persistent connections, instant connections are preferred due to the limited power of CIoT devices [6]. When the small sized data are transferred successfully, the connection is released immediately leading to longer delays. Hence, delay tolerant applications can use the CIoT services effortlessly. The channels are made severely competitive with concurrent access by concentrating a smaller amount of random-access channels (RACHs) with a large amount of connection requests over the CIoT systems. All CIoT devices may be accommodated effectively by alleviating the random-access procedure with instant congestion. The random access requests may undergo invalidation or collision due to the severe competition on the RACH. 
J. Sustain. Wireless Syst., vol. 02, no. 4, pp. 155-159

https://doi.org/10.36548/jsws.2020.4.003

\section{Literature Review}

The 3GPP standardization renders specific requirements for various service scenarios supported by the CIoT systems [7]. Simple operations are performed by the simplified design of the CIoT device hardware structure that enables deployment of huge number of devices at reduced cost. The power consumption is reduced by the CIoT devices. This ensures lesser maintenance and long operation time of the deployed devices. Two AA batteries can be used for achieving a lifetime of over ten years in the CIoT devices using existing standardizations. When compared to the conventional cellular networks, the better coverage is provided by the CIoT systems that extends to $20 \mathrm{~dB}$ [2]. Despite being positioned in unreachable and inaccessible regions, every CIoT device must be accommodated in the extended coverage. When compared to the conventional cellular networks, the CIoT systems have several distinguished special and unique characteristics. CIoT devices are also referred to as sensors in 3GPP technology [4]. The position at which these devices were deployed initially will be fixed and does not change for the CIoT devices except for some rare movement.

With respect to the pre-defined cycle, the data that is measured is reported to the network infrequently by these devices. Characteristics, similar to the ones originated from the devices is reflected in the CIoT services [5]. Instead of a real-time mobility management operation, a simple location update process can be provided by the CIoT systems. Based on the channel condition, the connected devices are managed effectively by the CIoT system with the coverage level concept. Coverage class, a CL concept has been adopted by several NB-CIoT technologies that are GSM based such as NB-OFDMA, NB-M2M and EC-GSM. The IoT network coverage extension can be enabled at a low cost with the help of NB-IoT standardization and 3GPP along with the concept of CL [8]. Based on the scenario, the signal-to-interference-plus-noise ratio (SINR) and received signal strength (RSS), categorization of CIoT devices is performed into various groups. The data transmissions and initial accesses are performed based on various parameters attached to each group of CIoT devices. Transmission power, number of transmission repeats, modulation and coding schemes (MCS) and other such parameter values must be compensated for the deteriorated channel. Despite operation under poor system coverage, differentiation of the CIoT devices offers more data channel environment and higher control level [7].

\section{Proposed Analysis Model}

The evolved Node B (eNodeB) is connected with a huge number of devices in the 3GPP CIoT system through random-access competition. When compared to the conventional devices, the NPRACH has larger amount of random-access requests for transmission of small-sized data that require repeated establishment of connections by the CIoT devices. Preambles can be used for distinguishing the NPRACH random access request for establishment of connections [8-9]. All CIoT devices send several random-access requests that cannot be accommodated by the limited number of identifiers in the preambles of the 3GPP. In order to obtain a stable random access, the simultaneous loading of the random-access requests must be reduced considerably at the NPRACH. Figure 1 provides the basic structure of a power efficient CL based time division random access scheme (TD-RAS). Multiple coverage levels with multiple CIoT devices is depicted and time slots are divided [10-11].

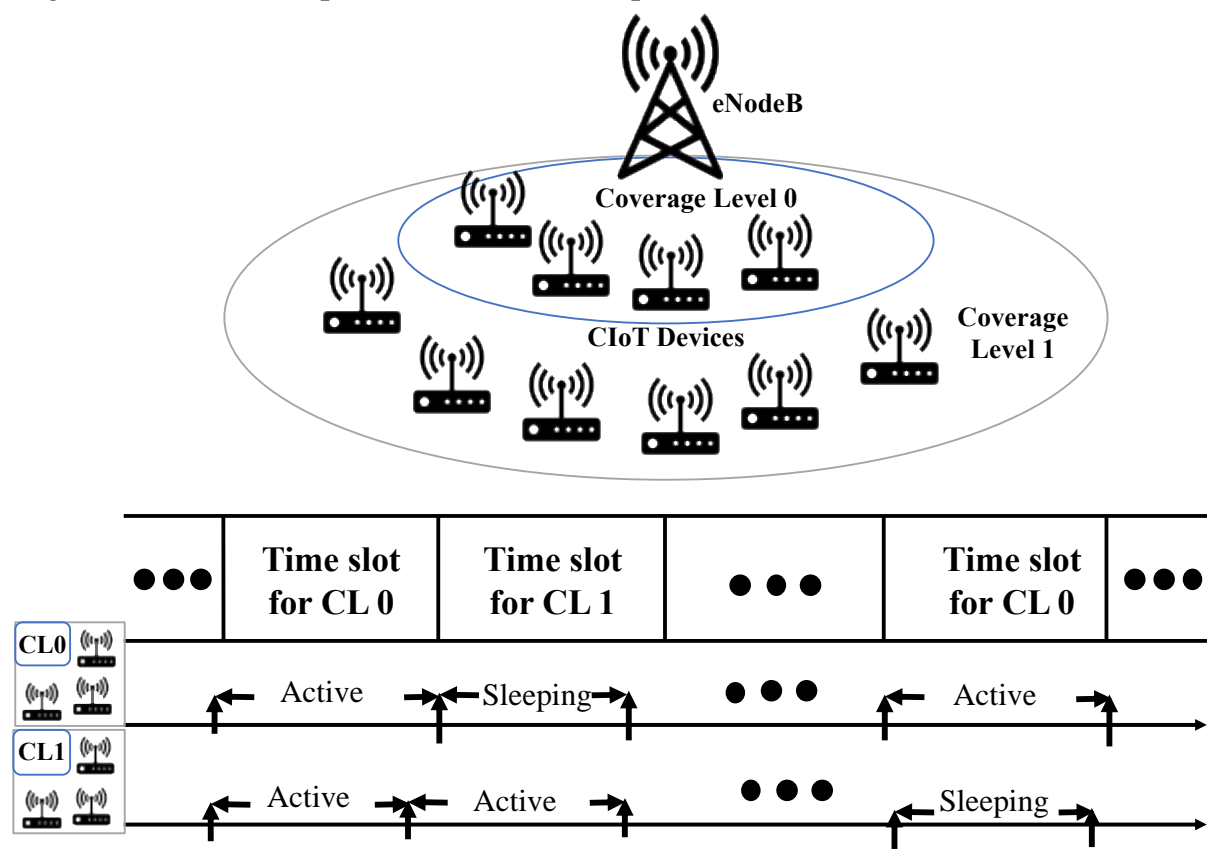

Figure 1. Power efficient CL based TD-RAS 
The performance evaluation of the proposed scheme can be done mathematically using Markov chain technique. The device behavior and instant conditions are represented by the transition probabilities and Markovian states [12-14]. Consider the coverage levels to be a, b and c for each Markovian state, the probability of collision of a transmitted preamble with that of other devices with similar CL can be represented by

$$
\mathrm{P}_{\mathrm{C}}=\mathrm{W}[\mathrm{P}(\mathrm{a}, \mathrm{b}+1, \mathrm{c} \mid \mathrm{a}, \mathrm{b}, 0)\}-------[1]
$$

Where $\mathrm{W}$ is the size of the back-off window. The switching or state transition time of the deive can be represented by

$$
\mathrm{T}_{\text {trans }}=\mathrm{N}_{\mathrm{CL}} \cdot \mathrm{T}------[2]
$$

where The total coverage levels is represented by $\mathrm{N}_{\mathrm{CL}}$ and the duration of random access is given by $\mathrm{T}$. The device access probability $\mathrm{P}_{\text {access }}$ is given by

$$
\mathrm{P}_{\mathrm{access}}=\alpha_{\mathrm{a}} \cdot\left(1-\mathrm{P}_{\mathrm{C}}\right)------[3]
$$

Where $\alpha_{a}=\sum_{b=0}^{j} \gamma_{a, b, c}---[4]$

$\gamma_{a, b, c}$ is the steady state probability of $(a, b, c)$. Similarly the $\mathrm{P}_{\mathrm{access}}$ and $\mathrm{N}_{\text {trans }}$ expressions can be obtained. The time lapse between the transmission and acknowledgement of the preamble can be represented by $\mathrm{T}_{\text {lap }}$.

\section{Simulation and Results}

The proposed scheme and conventional techniques are compared for performance evaluation for different values of CL at different time periods. Conventional scheme, conventional scheme with access class and an access probability of 0.5 , multiple coverage levels of 4 and 8 are compared for performance in terms of $P_{\text {trans }}, P_{\text {access }}$, $\mathrm{N}_{\text {trans }}, \mathrm{N}_{\text {access }}$ and $\mathrm{T}_{\text {lap }}$ is represented graphically in Figure 2, 3 and 4. Lines and symbols are used for representing the simulation and analytical model for the purpose of evaluating the results. Fixed-point iteration scheme is used for calculation of analytical results. Convergence is reached by iterative calculation of the analytical results. $\mathrm{C}++$ language is used for creating a self-coded event-based simulator that helps in obtaining the simulated results. Independent stationary point scheme is deployed for simulation and analysis considering the CIoT devices attached to the nearest eNodeB. The own event of the device is processed first until which no additional events are considered. Poisson distribution is used for representing the arrival event of CIoT devices in random access process initialization.

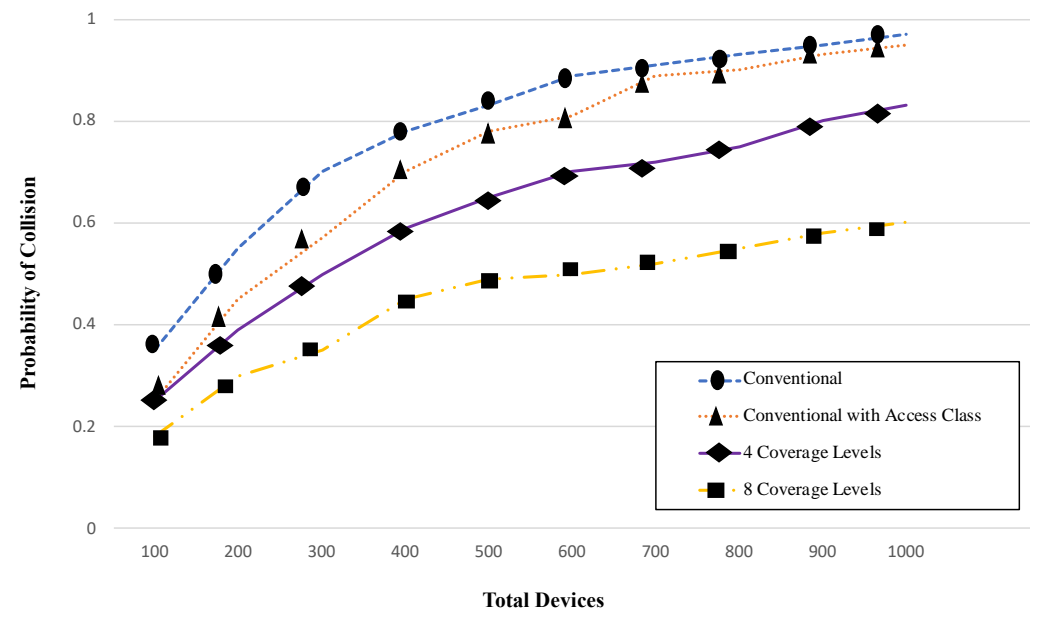

Figure 2: Collision probability of random-access failure $\mathrm{P}_{\mathrm{C}}$

Figure 2 represents the collision probability of random-access failure among that preambles that are transmitted. Figure 3 represents the analysis of $\mathrm{N}_{\text {access }}$ where a specific random-access duration is considered and the total CIoT devices realizing the random-access process positively are considered. Comparison of the conventional and propose systems provide dissimilar $\mathrm{N}_{\text {access }}$ values and maximum levels. Severe variations in increase and decrease of the values can be noticed in the parameter. Variation in the population also affects the $\mathrm{N}_{\text {access }}$ parameter. Using 8 CLs can help in achieving maximum $\mathrm{N}_{\text {access }}$ with the increase in the number of devices in the environment. 


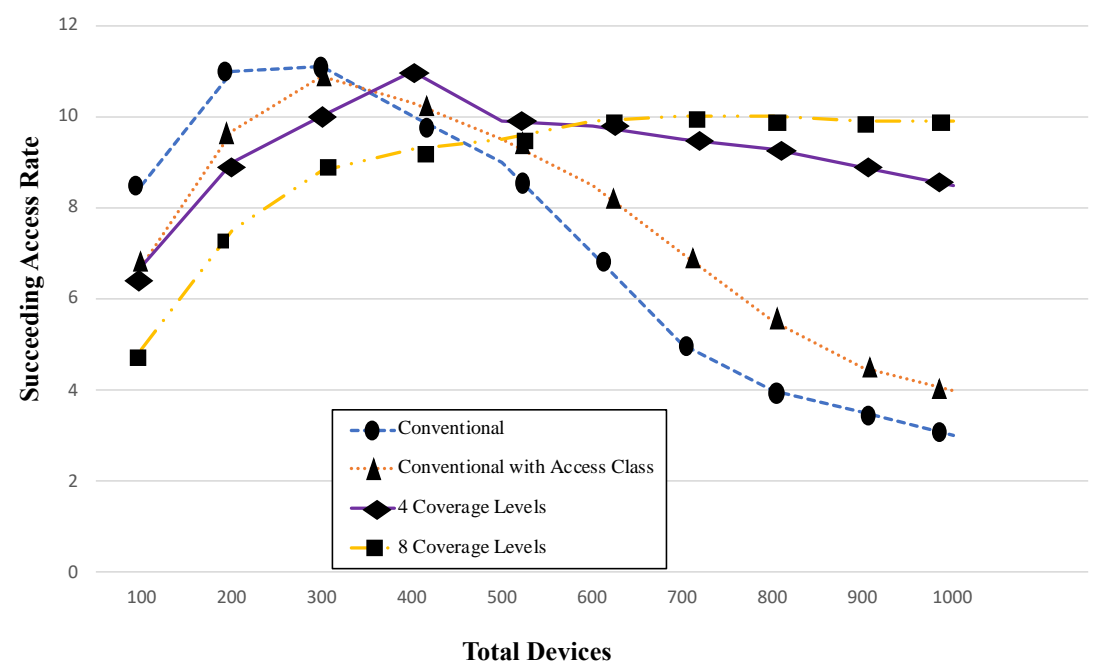

Figure 3: Graphical representation of $\mathrm{N}_{\text {access }}$ Analysis

Figure 4 represents $T_{\text {lap }}$, which is the average access delay representing the duration between transmission and acknowledgement of the first preamble. As the number of CIoT devices increase, $\mathrm{T}_{\text {lap }}$ increases. However, when largely populated CIoT devices are considered, $\mathrm{T}_{\text {lap }}$ increases at a slower rate as blocked random-access requests are not considered during calculation. All the crucial performance parameters are improved using the proposed scheme when compared to the conventional techniques. All operation should be held by the CIoT device that belongs to specific CL for the duration of activation of other CLs. When several segmented coverage levels are measured, the proposed scheme offers improved $\mathrm{T}_{\text {lap }}$ value. The performance of the CIoT devices are improved significantly along with the delay tolerance. This improves the tolerance of slight variations in $\mathrm{T}_{\text {lap }}$.

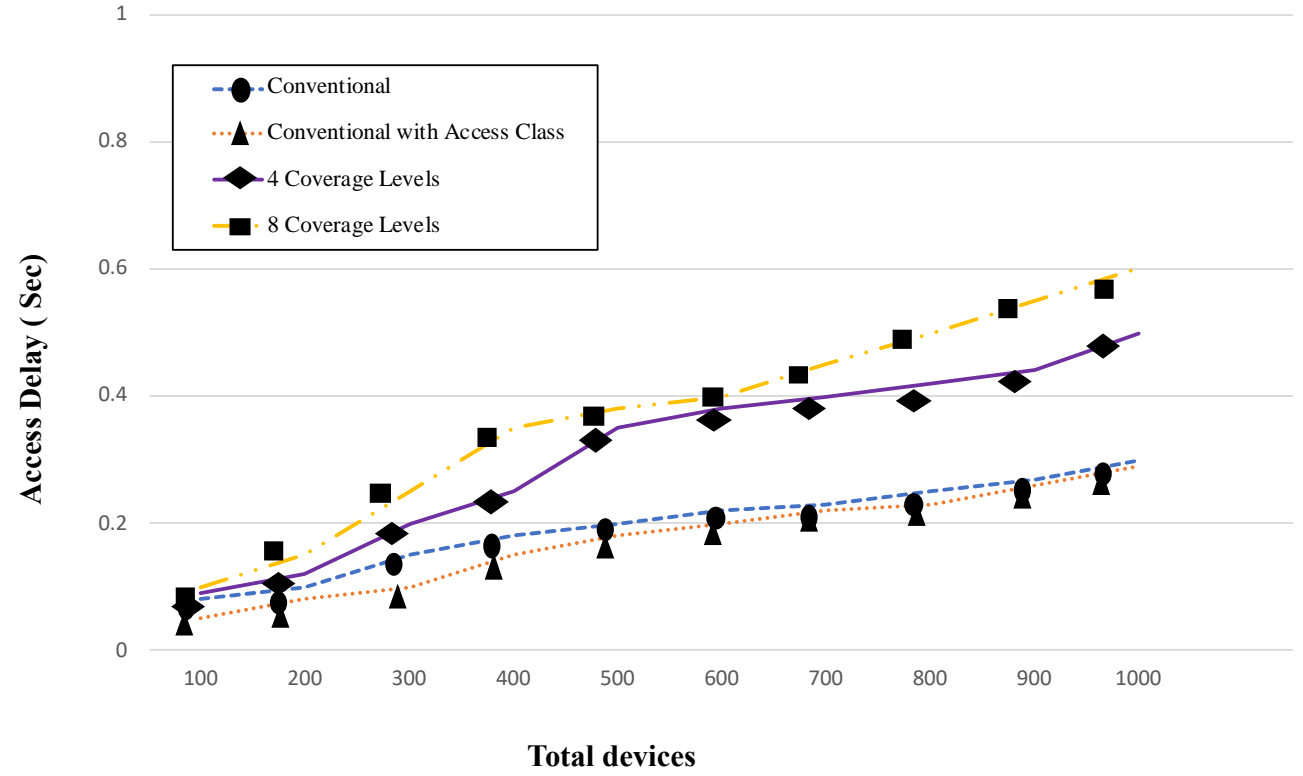

Figure 4: Average access delay $\mathrm{T}_{\text {lap }}$

\section{Conclusion}

The CIoT device coverage levels are considered under a power efficient time-division random-access model in this paper. Multiple coverage levels are used and the CIoT devices are assigned different time periods for performing he random-access process efficiently. Alleviation of momentary contention levels can be performed by distributing the concentrated random access loads into different time periods. Huge quantity of CIoT devices can be accommodated efficiently using the proposed scheme. Even when the device population is crowded, the collision probability of the proposed scheme is reduced by a considerable amount as demonstrated in the simulation and analysis results. High accuracy is obtained while estimating the performance of the proposed scheme with the analysis model. The probability of blocking access, success rate and collision control are 
enhanced by a significant level using this model in comparison to the conventional schemes. Future work is proposed at increasing the number of coverage levels further and the time slots for each coverage level while maintaining the system efficiency and power level even with large number of devices.

\section{References}

[1] Ergen, S. C., \& Varaiya, P. (2006). PEDAMACS: Power efficient and delay aware medium access protocol for sensor networks. IEEE Transactions on Mobile Computing, 5(7), 920-930.

[2] Ali, M. S., Hossain, E., \& Kim, D. I. (2017). LTE/LTE-A random access for massive machine-type communications in smart cities. IEEE Communications Magazine, 55(1), 76-83.

[3] Mehta, R. (2021). Throughput and resource optimization for adaptive coding-based random access networks with correlated sources. International Journal of Communication Systems, 34(1), e4673.

[4] Seo, J. B., Jung, B. C., \& Jin, H. (2018). Nonorthogonal random access for 5G mobile communication systems. IEEE Transactions on Vehicular Technology, 67(8), 7867-7871.

[5] Wu, Y., Gao, X., Zhou, S., Yang, W., Polyanskiy, Y., \& Caire, G. (2020). Massive Access for Future Wireless Communication Systems. IEEE Wireless Communications.

[6] De Gaudenzi, R., Del Rio Herrero, O., Gallinaro, G., Cioni, S., \& Arapoglou, P. D. (2018). Random access schemes for satellite networks, from VSAT to M2M: a survey. International Journal of Satellite Communications and Networking, 36(1), 66-107.

[7] Yu, H., Zou, J., \& Xu, C. (2018). Power-Efficient Random Access Design for Machine Type Communication. Electronics, 7(11), 286.

[8] Solt, F., Benarrouch, R., Tochou, G., Facklam, O., Frappé, A., Cathelin, A., ... \& Rabaey, J. M. (2020). Energy Efficient Heartbeat-Based MAC Protocol for WBAN Employing Body Coupled Communication. IEEE Access, 8, 182966-182983.

[9] Lin, H., \& Shin, W. Y. (2017). Non-orthogonal random access in MIMO cognitive radio networks: beamforming, power allocation, and opportunistic transmission. PloS one, 12(1), e0169902.

[10] Ghosh, A., Maeder, A., Baker, M., \& Chandramouli, D. (2019). 5G evolution: A view on 5G cellular technology beyond 3GPP release 15. IEEE Access, 7, 127639-127651.

[11] Höyhtyä, M., Apilo, O., \& Lasanen, M. (2018). Review of latest advances in 3GPP standardization: D2D communication in $5 \mathrm{G}$ systems and its energy consumption models. Future Internet, 10(1), 3.

[12] Beyene, Y. D., Jäntti, R., \& Ruttik, K. (2017). Random access scheme for sporadic users in 5G. IEEE Transactions on Wireless Communications, 16(3), 1823-1833.

[13] Valanarasu, M. R., \& Christy, A. (2019). Comprehensive Survey of Wireless Cognitive and 5G Networks. Journal of Ubiquitous Computing and Communication Technologies (UCCT), 23-32.

[14] Chen, J. I. Z. 5G Systems with Low Density Parity Check based Chanel Coding for Enhanced Mobile Broadband Scheme. 